\title{
UNIQUENESS OF GRADIENT RICCI SOLITONS
}

\author{
Simon BREndLE
}

\begin{abstract}
We show that a three-dimensional steady gradient Ricci soliton which is asymptotic to the Bryant soliton in a suitable sense must be isometric to the Bryant soliton.
\end{abstract}

\section{Introduction}

The Ricci flow, introduced by R. Hamilton [12] in 1982, has been studied intensively in recent years. In particular, the Ricci flow plays a key role in Perelman's proof of the Poincaré conjecture (cf. [16], [17], [18]). The Ricci flow also features prominently in the proof of the Differentiable Sphere Theorem for pointwise 1/4-pinched manifolds (cf. [1], [3], [4], [5]). For an introduction to Ricci flow, see e.g. [2] or [21].

In this paper, we are interested in self-similar solutions to the Ricci flow. Such solutions are referred to as Ricci solitons, and were first studied by Hamilton [13]. Recall that a Riemannian manifold $(M, g)$ is called a steady Ricci soliton if

$$
\text { Ric }+\frac{1}{2} \mathscr{L}_{\xi}(g)=0
$$

for some vector field $\xi$. Moreover, if $\xi=-\nabla f$ for some smooth function $f: M \rightarrow \mathbb{R}$, then $(M, g)$ is referred to as a steady gradient Ricci soliton. Ricci solitons play a fundamental role in the formation of singularities, and have been studied by many authors; see [8] for a survey.

The simplest example of a steady Ricci soliton is the so-called cigar soliton in dimension 2. The cigar soliton is rotationally symmetric, has positive Gaussian curvature, and is asymptotic to a cylinder near infinity. R. Bryant [6] has constructed an example of a steady gradient Ricci soliton in dimension 3. This solution is rotationally symmetric and has positive sectional curvature. Bryant's construction can be adapted to higher dimensions. In fact, for each $n \geq 3$, there exists an $n$-dimensional steady gradient Ricci soliton, which is rotationally symmetric and has positive curvature operator. This will be referred to as the Bryant soliton. Further examples of steady Ricci solitons were constructed by H.D. Cao [7] and T. Ivey [15].

It was shown by Hamilton that any two-dimensional gradient soliton is isometric to the cigar soliton up to scaling. In [16], G. Perelman conjectured a similar uniqueness property in dimension 3 :

Conjecture (G. Perelman [16]). Any three-dimensional steady gradient Ricci soliton with positive sectional curvature which satisfies a non-collapsing assumption at infinity is isometric to the Bryant soliton up to scaling.

Received by the editors November 12, 2010.

The author was supported in part by the National Science Foundation under grant DMS-0905628. 
We note that H. Guo [11] has obtained interesting results on the asymptotic geometry of a Ricci soliton near infinity. In a recent paper [9], H.D. Cao and Q. Chen proved uniqueness under the additional assumption that $(M, g)$ is locally conformally flat. The same result was proved independently by Catino and Mantegazza [10] under the assumption that $n \geq 4$.

Theorem 1 (H.D. Cao, Q. Chen [9]). Let $(M, g)$ be a steady gradient Ricci soliton of dimension $n \geq 3$. If $(M, g)$ is locally conformally flat, then $(M, g)$ is either flat or rotationally symmetric.

Throughout this paper, we will assume that $(M, g)$ is a three-dimensional steady gradient Ricci soliton. We will show that $(M, g)$ is rotationally symmetric, provided that $(M, g)$ satisfies certain asymptotic conditions near infinity. To that end, we fix a smooth function $\psi:(0,1) \rightarrow \mathbb{R}$ so that $\nabla R+\psi(R) \nabla f=0$ on the Bryant soliton. Moreover, we define

$$
u(s)=\log \psi(s)+\int_{\frac{1}{2}}^{s}\left(\frac{3}{2(1-t)}-\frac{1}{(1-t) \psi(t)}\right) d t .
$$

Then we have the following result:

Theorem 2. Let $(M, g)$ be a three-dimensional steady Ricci soliton. Suppose that the scalar curvature of $(M, g)$ is positive and approaches zero at infinity. Moreover, we assume that there exists an exhaustion of $M$ by bounded domains $\Omega_{l}$ such that

$$
\lim _{l \rightarrow \infty} \int_{\partial \Omega_{l}} e^{u(R)}\langle\nabla R+\psi(R) \nabla f, \nu\rangle=0 .
$$

Then $(M, g)$ is rotationally symmetric.

The proof of Theorem 2 is inspired in part by D.C. Robinson's proof of the uniqueness of the Schwarzschild black hole (cf. [14] and [20]).

The author would like to thank Fernando Marques for discussions. He is grateful to the referee for many useful comments.

\section{Some useful identities}

Let $(M, g)$ be a three-dimensional steady gradient Ricci soliton, so that Ric $=D^{2} f$ for some real-valued function $f$. We first collect some well-known facts:

Proposition 3. We have

$$
\partial_{i} R=-2 \operatorname{Ric}_{i j} \partial_{j} f
$$

and

$$
\Delta R+2|\mathrm{Ric}|^{2}=-\langle\nabla f, \nabla R\rangle .
$$

Proof. Using the contracted second Bianchi identity, we obtain

$$
\begin{aligned}
0 & =\partial_{i} R-2 g^{k l} D_{i} \operatorname{Ric}_{k l}+2 g^{k l} D_{k} \operatorname{Ric}_{i l} \\
& =\partial_{i} R-2 g^{k l} D_{i, k, l}^{3} f+2 g^{k l} D_{k, i, l}^{3} f \\
& =\partial_{i} R+2 g^{k l} R_{i k j l} \partial^{j} f \\
& =\partial_{i} R+2 \operatorname{Ric}_{i j} \partial^{j} f .
\end{aligned}
$$


This proves (2). To prove (3), we take the divergence on both sides of the previous identity. This yields

$$
\begin{aligned}
0 & =\Delta R+2 D^{i} \operatorname{Ric}_{i j} \partial^{j} f+2|\operatorname{Ric}|^{2} \\
& =\Delta R+\partial_{j} R \partial^{j} f+2|\operatorname{Ric}|^{2}
\end{aligned}
$$

as claimed.

It follows from (2) that the sum $R+|\nabla f|^{2}$ is constant. By scaling, we may ssume that $R+|\nabla f|^{2}=1$ at each point in $M$. We next define a tensor $B_{i j k}$ by

$$
\begin{aligned}
B_{i j k} & =\operatorname{Ric}_{i k} \partial_{j} f-\operatorname{Ric}_{i j} \partial_{k} f \\
& -\frac{1}{4}\left(\left(\partial_{j} R+2 R \partial_{j} f\right) g_{i k}-\left(\partial_{k} R+2 R \partial_{k} f\right) g_{i j}\right) .
\end{aligned}
$$

Note that the tensor $B_{i j k}$ vanishes on the set $\{R=1\}$.

It was shown by Cao and Chen [9] that the tensor $B_{i j k}$ agrees with the Cotten tensor of $(M, g)$, up to a constant factor. In particular, we have $B_{i j k}=0$ on the Bryant soliton.

Proposition 4. If $(M, g)$ is a steady gradient Ricci soliton, then we have

$$
\begin{aligned}
|B|^{2} & =-(1-R) \Delta R-\frac{3}{4}|\nabla R|^{2} \\
& -\langle\nabla f, \nabla R\rangle-R^{2}(1-R) .
\end{aligned}
$$

Proof. Using (2) and (3), we obtain

$$
\begin{aligned}
& \sum_{i, j, k}\left|B_{i j k}\right|^{2} \\
& =\sum_{i, j, k}\left|\operatorname{Ric}_{i k} \partial_{j} f-\operatorname{Ric}_{i j} \partial_{k} f\right|^{2}+\frac{1}{4}|\nabla R+2 R \nabla f|^{2} \\
& -\sum_{i, j, k}\left(\operatorname{Ric}_{i k} \partial_{j} f-\operatorname{Ric}_{i j} \partial_{k} f\right)\left(\partial^{j} R+2 R \partial^{j} f\right) g^{i k} \\
& =\sum_{i, j, k}\left|\operatorname{Ric}_{i k} \partial_{j} f-\operatorname{Ric}_{i j} \partial_{k} f\right|^{2}-\frac{1}{4}|\nabla R+2 R \nabla f|^{2} \\
& =\left.2\left|\operatorname{Ric}^{2}\right| \nabla f\right|^{2}-2 \sum_{i, j, k} \operatorname{Ric}_{i j} \partial_{j} f \operatorname{Ric}_{i k} \partial_{k} f-\frac{1}{4}|\nabla R+2 R \nabla f|^{2} \\
& =\left.2\left|\operatorname{Ric}^{2}\right| \nabla f\right|^{2}-\frac{1}{2}|\nabla R|^{2}-\frac{1}{4}|\nabla R+2 R \nabla f|^{2} \\
& =-(\Delta R+\langle\nabla f, \nabla R\rangle)|\nabla f|^{2}-\frac{1}{2}|\nabla R|^{2}-\frac{1}{4}|\nabla R+2 R \nabla f|^{2} \\
& =-|\nabla f|^{2} \Delta R-|\nabla f|^{2}\langle\nabla f, \nabla R\rangle-\frac{3}{4}|\nabla R|^{2} \\
& -R\langle\nabla f, \nabla R\rangle-R^{2}|\nabla f|^{2} .
\end{aligned}
$$


Using the identity $|\nabla f|^{2}=1-R$, we conclude that

$$
\begin{aligned}
|B|^{2} & =-(1-R) \Delta R-\frac{3}{4}|\nabla R|^{2} \\
& -\langle\nabla f, \nabla R\rangle-R^{2}(1-R),
\end{aligned}
$$

as claimed.

In the next step, we choose a smooth function $\psi:(0,1) \rightarrow \mathbb{R}$ such that $\nabla R+$ $\psi(R) \nabla f=0$ on the Bryant soliton.

Proposition 5. If $(M, g)$ is a steady gradient Ricci soliton, then the vector field $X=\nabla R+\psi(R) \nabla f$ satisfies

$$
\begin{aligned}
& (1-R) \operatorname{div} X \\
& =-|B|^{2}-\frac{3}{4}\langle\nabla R-\psi(R) \nabla f, X\rangle-\langle\nabla f, X\rangle \\
& +(1-R) \psi^{\prime}(R)\langle\nabla f, X\rangle-\frac{3}{4}(1-R) \psi(R)^{2}+(1-R) \psi(R) \\
& -R^{2}(1-R)+R(1-R) \psi(R)-(1-R)^{2} \psi(R) \psi^{\prime}(R) .
\end{aligned}
$$

Proof. Using (4), we obtain

$$
\begin{aligned}
& (1-R) \operatorname{div} X \\
& =(1-R) \Delta R+(1-R) \psi(R) \Delta f+(1-R) \psi^{\prime}(R)\langle\nabla f, \nabla R\rangle \\
& =-|B|^{2}-\frac{3}{4}|\nabla R|^{2}-\langle\nabla f, \nabla R\rangle-R^{2}(1-R) \\
& +(1-R) \psi(R) \Delta f+(1-R) \psi^{\prime}(R)\langle\nabla f, \nabla R\rangle \\
& =-|B|^{2}-\frac{3}{4} \psi(R)^{2}|\nabla f|^{2}-\frac{3}{4}\langle\nabla R-\psi(R) \nabla f, X\rangle \\
& -\langle\nabla f, X\rangle+\psi(R)|\nabla f|^{2}-R^{2}(1-R)+(1-R) \psi(R) \Delta f \\
& +(1-R) \psi^{\prime}(R)\langle\nabla f, X\rangle-(1-R) \psi(R) \psi^{\prime}(R)|\nabla f|^{2} \\
& =-|B|^{2}-\frac{3}{4}(1-R) \psi(R)^{2}-\frac{3}{4}\langle\nabla R-\psi(R) \nabla f, X\rangle \\
& -\langle\nabla f, X\rangle+(1-R) \psi(R)-R^{2}(1-R)+R(1-R) \psi(R) \\
& +(1-R) \psi^{\prime}(R)\langle\nabla f, X\rangle-(1-R)^{2} \psi(R) \psi^{\prime}(R) .
\end{aligned}
$$

This proves the assertion.

Corollary 6. The function $\psi$ satisfies the differential equation

$$
0=-\frac{3}{4} \psi(s)^{2}+\psi(s)-s^{2}+s \psi(s)-(1-s) \psi(s) \psi^{\prime}(s)
$$

for all $s \in(0,1)$.

Proof. The identity (5) holds for any steady gradient Ricci soliton. In particular, it holds for the Bryant soliton. On the other hand, we have $B=0$ and $X=0$ on the Bryant soliton. From this the assertion follows. 
Proposition 7. Assume that $\psi$ is chosen so that $\nabla R+\psi(R) \nabla f=0$ on the Bryant soliton. Then

$$
\begin{aligned}
(1-R) \operatorname{div} X & =-|B|^{2}-\frac{3}{4}\langle\nabla R-\psi(R) \nabla f, X\rangle \\
& -\langle\nabla f, X\rangle+(1-R) \psi^{\prime}(R)\langle\nabla f, X\rangle .
\end{aligned}
$$

Proof. This follows immediately from (5) and (6).

In the next step, we consider the function

$$
u(s)=\log \psi(s)+\int_{\frac{1}{2}}^{s}\left(\frac{3}{2(1-t)}-\frac{1}{(1-t) \psi(t)}\right) d t .
$$

Proposition 8. We have

$$
(1-R) e^{-u(R)} \operatorname{div}\left(e^{u(R)} X\right)=-|B|^{2}-\frac{R(R-\psi(R))}{\psi(R)^{2}}|X|^{2} .
$$

Proof. Using (7), we obtain

$$
\begin{aligned}
& (1-R) e^{-u(R)} \operatorname{div}\left(e^{u(R)} X\right) \\
& =(1-R) \operatorname{div} X+(1-R) u^{\prime}(R)\langle\nabla R, X\rangle \\
& =-|B|^{2}-\frac{3}{4}\langle\nabla R-\psi(R) \nabla f, X\rangle-\langle\nabla f, X\rangle+(1-R) \psi^{\prime}(R)\langle\nabla f, X\rangle \\
& +\frac{3}{2}\langle\nabla R, X\rangle-\frac{1}{\psi(R)}\langle\nabla R, X\rangle+(1-R) \frac{\psi^{\prime}(R)}{\psi(R)}\langle\nabla R, X\rangle \\
& =-|B|^{2}+\frac{3}{4}\langle\nabla R+\psi(R) \nabla f, X\rangle-\frac{1}{\psi(R)}\langle\nabla R+\psi(R) \nabla f, X\rangle \\
& +(1-R) \frac{\psi^{\prime}(R)}{\psi(R)}\langle\nabla R+\psi(R) \nabla f, X\rangle \\
& =-|B|^{2}+\frac{3}{4}|X|^{2}-\frac{1}{\psi(R)}|X|^{2}+(1-R) \frac{\psi^{\prime}(R)}{\psi(R)}|X|^{2} .
\end{aligned}
$$

Using (6), we obtain

$$
-\frac{s(s-\psi(s))}{\psi(s)^{2}}=\frac{3}{4}-\frac{1}{\psi(s)}+(1-s) \frac{\psi^{\prime}(s)}{\psi(s)} .
$$

Putting these facts together, the assertion follows.

\section{Proof of the Theorem 2}

Lemma 9. For $s \rightarrow 1$, we have $\psi(s)=\frac{2}{3}+O(\sqrt{1-s})$.

Proof. On the Bryant soliton, we have

$$
0=\partial_{i} R+\psi(R) \partial_{i} f=-2 \sum_{i, j} \operatorname{Ric}_{i j} \partial_{j} f+\psi(R) \partial_{i} f .
$$

Therefore, the vector $\nabla f$ is an eigenvector of the Ricci tensor with eigenvalue $\frac{\psi(R)}{2}$. On the other hand, we have $\operatorname{Ric}_{i j}=\frac{1}{3} g_{i j}+O(|x|)$ near the origin. This implies $\frac{\psi(R(x))}{2}=\frac{1}{3}+O(|x|)$ near the origin. From this, the assertion follows easily. 
Lemma 10. The limit $\lim _{s \rightarrow 1} u(s)$ exists.

Proof. It follows from Lemma 9 that

$$
\frac{1}{1-s}\left(\frac{3}{2}-\frac{1}{\psi(s)}\right)=O\left(\frac{1}{\sqrt{1-s}}\right)
$$

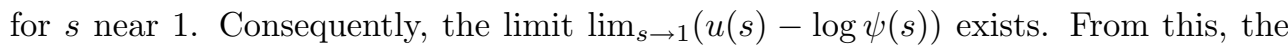
assertion follows.

Proposition 11. We have $\psi(s)<s$ for all $s \in(0,1)$.

Proof. Suppose that the assertion is false. Let

$$
s_{0}=\sup \{s \in(0,1): \psi(s) \geq s\} .
$$

Since $\lim _{s \rightarrow 1} \psi(s)=\frac{2}{3}$, we conclude that $s_{0} \in(0,1)$. Moreover, we have $\psi\left(s_{0}\right)=s_{0}$ and $\psi^{\prime}\left(s_{0}\right) \leq 1$. Using $(9)$, we obtain

$$
\begin{aligned}
0 & =-\frac{s_{0}\left(s_{0}-\psi\left(s_{0}\right)\right)}{\psi\left(s_{0}\right)^{2}} \\
& =\frac{3}{4}-\frac{1}{\psi\left(s_{0}\right)}+\left(1-s_{0}\right) \frac{\psi^{\prime}\left(s_{0}\right)}{\psi\left(s_{0}\right)} \\
& \leq \frac{3}{4}-\frac{1}{s_{0}}+\frac{1-s_{0}}{s_{0}} \\
& =-\frac{1}{4} .
\end{aligned}
$$

This is a contradiction.

Proposition 12. Let $\Omega$ be a bounded domain in $M$ with smooth boundary. Moreover, suppose that $R<1$ at each point on $\partial \Omega$. Then

$$
\int_{\Omega \cap\{R<1\}} \frac{e^{u(R)}}{1-R}|B|^{2} \leq-\int_{\partial \Omega} e^{u(R)}\langle X, \nu\rangle .
$$

Proof. Let us fix a smooth cut-off function $\chi:[0, \infty) \rightarrow[0,1]$ such that $\chi(s)=0$ for $s \leq 1$ and $\chi(s)=1$ for $s \geq 2$. Moreover, let $\chi_{\varepsilon}(s)=\chi\left(\frac{s}{\varepsilon}\right)$. It follows from Proposition 11 and (8) that

$$
(1-R) e^{-u(R)} \operatorname{div}\left(e^{u(R)} X\right) \leq-|B|^{2} .
$$

Using the divergence theorem, we obtain

$$
\begin{aligned}
\int_{\partial \Omega} \chi_{\varepsilon}(1-R) e^{u(R)}\langle X, \nu\rangle & =\int_{\Omega \cap\{R<1\}} \operatorname{div}\left(\chi_{\varepsilon}(1-R) e^{u(R)} X\right) \\
& =\int_{\Omega \cap\{R<1\}} \chi_{\varepsilon}(1-R) \operatorname{div}\left(e^{u(R)} X\right) \\
& -\int_{\Omega \cap\{R<1\}} \chi_{\varepsilon}^{\prime}(1-R) e^{u(R)}\langle X, \nabla R\rangle \\
& \leq-\int_{\Omega \cap\{R<1\}} \chi_{\varepsilon}(1-R) \frac{e^{u(R)}}{1-R}|B|^{2} \\
& -\int_{\Omega \cap\{R<1\}} \chi_{\varepsilon}^{\prime}(1-R) e^{u(R)}\langle X, \nabla R\rangle .
\end{aligned}
$$


We claim that

$$
\int_{\Omega \cap\{R<1\}} \chi_{\varepsilon}^{\prime}(1-R) e^{u(R)}\langle X, \nabla R\rangle \rightarrow 0
$$

as $\varepsilon \rightarrow 0$. Using the identity

$$
\langle X, \nabla R\rangle=4 \operatorname{Ric}_{i j} \operatorname{Ric}_{i k} \partial_{j} f \partial_{k} f-2 \psi(R) \operatorname{Ric}_{j k} \partial_{j} f \partial_{k} f,
$$

we obtain

$$
|\langle X, R\rangle| \leq C_{1}|\nabla f|^{2}=C_{1}(1-R)
$$

at each point on $\Omega$. Hence, on the set $\Omega \cap\{\varepsilon \leq 1-R \leq 2 \varepsilon\}$, we have

$$
\begin{aligned}
\chi_{\varepsilon}^{\prime}(1-R) e^{u(R)}|\langle X, \nabla R\rangle| & \leq C_{2} \varepsilon^{-1}|\langle X, \nabla R\rangle| \\
& \leq C_{1} C_{2} \varepsilon^{-1}(1-R) \\
& \leq 2 C_{1} C_{2} .
\end{aligned}
$$

Here, $C_{1}$ and $C_{2}$ are positive constants which may depend on $\Omega$, but not $\varepsilon$. This implies

$$
\left|\int_{\Omega \cap\{R<1\}} \chi_{\varepsilon}^{\prime}(1-R) e^{u(R)}\langle X, \nabla R\rangle\right| \leq 2 C_{1} C_{2} \operatorname{vol}(\{\varepsilon \leq 1-R \leq 2 \varepsilon\}),
$$

and the right hand side converges to 0 as $\varepsilon \rightarrow 0$. This proves (11). Combining (10) and (11), we conclude that

$$
\int_{\partial \Omega} e^{u(R)}\langle X, \nu\rangle \leq-\int_{\Omega \cap\{R<1\}} \frac{e^{u(R)}}{1-R}|B|^{2},
$$

as claimed.

We now complete the proof of Theorem 2. By assumption, we can find an exhaustion of $M$ by bounded domains $\Omega_{l}$ such that

$$
\lim _{l \rightarrow \infty} \int_{\partial \Omega_{l}} e^{u(R)}\langle X, \nu\rangle=0 .
$$

Using Proposition 12, we obtain

$$
\int_{\Omega_{l} \cap\{R<1\}} \frac{e^{u(R)}}{1-R}|B|^{2} \leq-\int_{\partial \Omega_{l}} e^{u(R)}\langle X, \nu\rangle .
$$

Passing to the limit as $l \rightarrow \infty$ gives

$$
\int_{\{R<1\}} \frac{e^{u(R)}}{1-R}|B|^{2}=0 .
$$

Therefore, the tensor $B$ vanishes on the set $\{R<1\}$. On the other hand, it is easy to see that the set $\{R<1\}$ is dense. Therefore, the tensor $B$ vanishes identically. It now follows from work of Cao and Chen $[9]$ that $(M, g)$ is rotationally symmetric. 


\section{References}

[1] S. Brendle, A general convergence result for the Ricci flow, Duke Math. J. 145, 585-601 (2008)

[2] S. Brendle, Ricci Flow and the Sphere Theorem, Graduate Studies in Mathematics, vol. 111, American Mathematical Society (2010)

[3] S. Brendle and R. Schoen, Manifolds with 1/4-pinched curvature are space forms, J. Amer. Math. Soc. 22, 287-307 (2009)

[4] S. Brendle and R. Schoen, Classification of manifolds with weakly 1/4-pinched curvatures, Acta Math. 200, 1-13 (2008)

[5] S. Brendle and R. Schoen, Curvature, sphere theorems, and the Ricci flow, Bulletin of the American Mathematical Society 48, 1-32 (2011)

[6] R.L. Bryant, Ricci flow solitons in dimension three with SO(3)-symmetries, available at www.math.duke.edu/ bryant/3DRotSymRicciSolitons.pdf

[7] H.D. Cao, Existence of gradient Kähler-Ricci solitons, Elliptic and Parabolic Methods in Geometry (Minneapolis, 1994), 1-16, A.K. Peters, Wellesley MA, 1996

[8] H.D. Cao, Recent progress on Ricci solitons, arxiv:0908.2006

[9] H.D. Cao and Q. Chen, On locally conformally flat gradient steady Ricci solitons, to appear in Trans. Amer. Math. Soc.

[10] G. Catino and C. Mantegazza, Evolution of the Weyl tensor under the Ricci flow under the Ricci flow, to appear in Annales de l'Institut Fourier

[11] H. Guo, Area growth rate of the level surface of the potential function on the 3-dimensional steady Ricci soliton, Proc. Amer. Math. Soc. 137, 2093-2097 (2009)

[12] R. Hamilton, Three-manifolds with positive Ricci curvature, J. Diff. Geom. 17, 255-306 (1982)

[13] R. Hamilton, The formation of singularities in the Ricci flow, Surveys in Differential Geometry, vol. II, 7-136, International Press, Somerville MA (1995)

[14] W. Israel, Event horizons in static vacuum space-times, Phys. Rev. 164, 1776-1779 (1967)

[15] T. Ivey, New examples of complete Ricci solitons, Proc. Amer. Math. Soc. 122, 241-245 (1994)

[16] G. Perelman, The entropy formula for the Ricci flow and its geometric applications, arxiv:0211159

[17] G. Perelman, Ricci flow with surgery on three-manifolds, arxiv:0303109

[18] G. Perelman, Finite extinction time for solutions to the Ricci flow on certain three-manifolds, arxiv:0307245

[19] P. Petersen and W. Wylie, On the classification of gradient Ricci solitons, arxiv:0712.1298

[20] D.C. Robinson, A simple proof of the generalization of Israel's theorem, General Relativity and Gravitation 8, 695-698 (1977)

[21] P. Topping, Lectures on the Ricci Flow, London Mathematical Society Lecture Notes Series, vol. 325, Cambridge University Press, Cambridge (2006)

Department of Mathematics, Stanford University, 450 Serra Mall, Bldg 380, Stanford, CA 94305 